Purdue University

Purdue e-Pubs

CTRC Research Publications

Cooling Technologies Research Center

2012

\title{
Thermodynamic and Kinetic Investigation of a Chemical Reaction-Based Miniature Heat Pump
}

Scott M. Flueckiger

Purdue University

Fabien Volle

Laboratoire des Sciences des Procédés et des Matériaux

S V. Garimella

Purdue University, sureshg@purdue.edu

Rajiv K. Mongia

Intel Corporation

Follow this and additional works at: http://docs.lib.purdue.edu/coolingpubs

Flueckiger, Scott M.; Volle, Fabien; Garimella, S V.; and Mongia, Rajiv K., "Thermodynamic and Kinetic Investigation of a Chemical Reaction-Based Miniature Heat Pump" (2012). CTRC Research Publications. Paper 182.

http://dx.doi.org/http://dx.doi.org/10.1016/j.enconman.2012.04.015

This document has been made available through Purdue e-Pubs, a service of the Purdue University Libraries. Please contact epubs@purdue.edu for additional information. 


\title{
Thermodynamic and Kinetic Investigation of a Chemical Reaction-Based Miniature Heat Pump*
}

\author{
Scott M. Flueckiger ${ }^{1}$, Fabien Volle ${ }^{2}$, Suresh V. Garimella ${ }^{1 * *}$, Rajiv K. Mongia ${ }^{3}$ \\ ${ }^{1}$ Cooling Technologies Research Center, an NSF I/UCRC \\ School of Mechanical Engineering and Birck Nanotechnology Center \\ 585 Purdue Mall, Purdue University \\ West Lafayette, Indiana 47907-2088 USA \\ ${ }^{2}$ Laboratoire des Sciences des Procédés et des Matériaux (LSPM, UPR 3407 CNRS), \\ Université Paris XIII, 99 avenue J. B. Clément, \\ 93430 Villetaneuse, France \\ ${ }^{3}$ Intel Corporation \\ Santa Clara, California 95054 USA
}

* Submitted for publication in Energy Conversion and Management

** Author to who correspondence should be addressed: (765) 494-5621, sureshg@purdue.edu 


\section{Abstract}

Representative reversible endothermic chemical reactions (paraldehyde depolymerization and 2-proponal dehydrogenation) are theoretically assessed for their use in a chemical heat pump design for compact thermal management applications. Equilibrium and dynamic simulations are undertaken to explore the operation of the heat pump which upgrades waste heat from near room temperature by approximately 20 degrees in a minimized system volume. A model is developed based on system mass and energy balances coupled with kinetic equations to ascertain mixture conditions at each state point in the loop, as well as mass flow rate, minimum work input, and minimum endothermic reactor volume according to defined reservoir temperatures and desired heat load. Assuming that a pervaporation process is employed in both reaction systems to achieve the requisite mixture compositions for sustained operation, the simulations show that the chemical heat pump can pump $5 \mathrm{~W}$ of power with endothermic reactor volumes of as little as 60

$\mathrm{cm}^{3}$ to $93 \mathrm{~cm}^{3}$, depending on the selected chemical reaction. Low exergy efficiencies remain a significant design consequence, but the system performance in terms of environmental impact and COP are comparable with, and in some cases better than, the performance of alternative technologies under the same conditions.

Keywords: Miniature chemical heat pump, thermal management, energy conversion, paraldehyde depolymerization, 2-propanol dehydrogenation 


\section{Nomenclature}

$a \quad$ Activity coefficient, -

C Mass concentration, $\mathrm{mol} / \mathrm{kg}$

F Molar flow rate, $\mathrm{mol} / \mathrm{s}$

$h \quad$ Specific enthalpy, $\mathrm{J} / \mathrm{mol}$

$K \quad$ Equilibrium constant, -

$n \quad$ Moles, mol

$P \quad$ Pressure, $\mathrm{kPa}$

$Q \quad$ Heat load, W

$r \quad$ Reaction rate, $\mathrm{mol} / \mathrm{m}^{3}-\mathrm{s}$

$s \quad$ Specific entropy, J/mol-K

$T \quad$ Temperature, $\mathrm{K}$

$x \quad$ Mass fraction, -

$X \quad$ Conversion, -

$y \quad$ Mole fraction, -

V Volume, $\mathrm{cm}^{3}$

$Z \quad$ Thermoelectric figure of merit, -

\section{Greek}

$\theta \quad$ Reaction conversion, -

$\rho \quad$ Density, $\mathrm{kg} / \mathrm{m}^{3}$

\section{Subscripts}

A Acetaldehyde

Ac Acetone

end Endothermic 


\begin{tabular}{ll} 
exo & Exothermic \\
$\mathrm{g}$ & Gaseous phase \\
$\mathrm{l}$ & Liquid phase \\
$\mathrm{Pa}$ & Paraldehyde \\
$\mathrm{H} 2$ & Hydrogen \\
$\mathrm{IPA}$ & 2-propanol \\
$\mathrm{r}$ & Reaction \\
sat & Saturation \\
$\mathrm{v}$ & Vaporization \\
w & Work \\
\multicolumn{2}{l}{ Acronyms }
\end{tabular}

COP Coefficient of Performance

CSTR Continuously Stirred Tank Reactor

PFR Plug Flow Reactor

\section{Introduction}

In accordance with the second law of thermodynamics, all thermal power systems discharge some quantity of heat to the surroundings, commonly designated as waste heat. Heat is also dissipated from most of the supplied electrical power in electronic and computer systems. There is significant interest in recovering these low-grade heat supplies at all design scales to improve thermal efficiencies and reduce operating costs. In addition, adequate heat removal strategies are essential for ensuring that the electronics operate at rated temperatures and thus maintain reliability and performance. While a rich variety of heat removal solutions have been investigated in the literature $[1,2]$, they rely on an ambient temperature that is lower than the 
system temperature, i.e., on rejecting heat to a lower-temperature air or liquid stream. There is a continuing need to develop approaches for removal of heat against a temperature gradient, i.e., from lower system temperatures to a higher-temperature ambient [3]. Heat pumps offer a viable option in such situations, as well as for waste heat recovery.

A heat pump upgrades heat from a low-temperature reservoir to a high-temperature reservoir (usually via work input), increasing the exergy or usefulness of the thermal energy. The most prevalent forms of heat pump technologies include mechanical and absorption heat pumps. A mechanical heat pump [4] is typically a vapor compression cycle in which the working fluid undergoes phase change (evaporation and condensation) to absorb and release heat between two thermal reservoirs. To maintain fluid transport in the system, work is input via a compressor upstream of the condenser.

In an absorption heat pump, a secondary fluid is introduced into the system to replace mechanical compression with pump work by absorbing the evaporated refrigerant. After exiting the evaporator, the refrigerant is mixed with the secondary fluid, yielding a two-component multi-phase mixture. This mixture is then pumped to a generator where the two fluids separate when subjected to high-temperature heat input; the refrigerant enters the condenser while the absorbent flows back to the absorber (completing the cycle). The most common refrigerantabsorbent combinations are ammonia-water and water-lithium bromide. Mumah et al. [5] present a procedure for the generation of properties that can be used for design, simulation and optimization of ammonia-water absorption systems. Such properties include enthalpy, vapor pressure, vapor concentration, specific heat capacity and entropy. Gupta et al. [6] compare three different cooling systems, including a $\mathrm{LiBr}-\mathrm{H}_{2} \mathrm{O}$ absorption system. They show through COP and refrigeration capacity calculations that this system has a high refrigeration capacity when operating with heat source temperatures below $90{ }^{\circ} \mathrm{C}$. Haywood et al. [7] report significant 
gains in energy efficiency for data centers with the inclusion of absorption chiller waste heat recovery. The solid sorption or adsorption heat pump developed and studied by Wang et al. [8] uses activated carbon-methanol and achieved $150 \mathrm{~W} / \mathrm{kg}$ of activated carbon for air conditioning with a COP of approximately 0.4. Gong et al. [9] obtain a COP of 0.43 for an adsorption chiller with a composite adsorbent material. A chemical heat pump, on the other hand, replaces the liquid-vapor phase change in a vapor compression cycle with a reversible endothermic chemical reaction to transport heat between the reservoir temperatures. By avoiding compression and additional system components associated with absorption cooling, the chemical heat pump design has potential for minimizing system volume, a feature that is highly advantageous for compact cooling applications. As shown in Figure 1, an idealized loop is composed of two plugflow reactors, a work input device, and an expansion valve. The chemical mixture first enters a low-temperature reactor (state 1) in which heat is absorbed by the endothermic reaction from the cold thermal reservoir. As with the other heat pump concepts, work input is needed to drive the fluid to the high-temperature reactor (state 2 to state 3 ), where heat is released via the reverse endothermic reaction. As the chemical reactions commonly involve liquid and vapor phases, a scroll device may be the most appropriate for compression of the two-phase mixture. The potential for use of an alternative ejector concept is discussed in Section 4.3. The expansion valve routes the product of the exothermic reactor (state 4) back to the endothermic reactor and closes the loop.

An extensive list of candidate reversible reactions has been proposed for chemical heat pumps [109], with the appropriate selection being dependent on the required reservoir temperatures in a particular application. For instance, a $\mathrm{CaO} / \mathrm{H}_{2} \mathrm{O} / \mathrm{Ca}(\mathrm{OH})_{2}$ chemical heat pump as described by Fujimoto et al. [11] can store thermal energy from industrial waste heat, solar heat and terrestrial heat in the form of chemical energy by an endothermic dehydration of 
$\mathrm{Ca}(\mathrm{OH})_{2}$, and release it at various temperature levels by an exothermic hydration of $\mathrm{CaO}$ when needed. This system could help address the increasing demand for energy storage and availability. Most of the systems considered in the literature concern operation at temperatures in the range of $350 \mathrm{~K}$ to $700 \mathrm{~K}$. However, thermal management applications in consumer electronics require lower operational temperatures on the order of $305 \mathrm{~K}$ to $322 \mathrm{~K}$. This temperature range creates additional challenges due to the reduced rates of reactions often associated with low temperatures and the small temperature difference between the reactors.

Of the available chemical reactions for heat pumping, paraldehyde $\left(\mathrm{C}_{6} \mathrm{H}_{12} \mathrm{O}_{3}\right)$ depolymerization and 2-propanol $\left(\left(\mathrm{CH}_{3}\right)_{2} \mathrm{CHOH}\right)$ dehydrogenation may both satisfy the required temperature range with acceptable chemical characteristics. Previous numerical studies of chemical heat pumps with paraldehyde depolymerization report coefficients of performance (COP) comparable to a vapor compression cycle [12]. In addition to thermodynamic performance, the size of the cooling system must also be considered to assess practical implementation. Past studies for paraldehyde depolymerization [12-14] have not considered cooling system size in their analysis.

In this work, the paraldehyde depolymerization and 2-propanol dehydrogenation reactions are separately investigated to assess their potential for chemical heat pump operation between $305 \mathrm{~K}$ and $322 \mathrm{~K}$, a typical temperature range encountered in electronics. Previous studies of 2-proponal dehydrogenation [15-18] were focused on higher reservoir temperatures. Constraints on reactant conversion enforced by chemical equilibrium are taken into account. In both studies, the default energy transport at the endothermic reactor is $5 \mathrm{~W}$. From detailed thermodynamic and kinetic analyses of the reversible reactions, required reactor volumes are estimated to determine viability as cooling solutions in consumer electronics for the default heat load. Viability is also assessed through comparison with other existing technologies. 


\section{Method and Materials}

Separate investigations for the paraldehyde depolymerization and the 2-propanol dehydrogenation are described in detail in this section.

\subsection{Paraldehyde Depolymerization}

Liquid paraldehyde (2, 4, 6-trimethyl-1, 3, 5-trioxane) reversibly depolymerizes to liquid acetaldehyde $\left(\mathrm{CH}_{3} \mathrm{CHO}\right)$ when in contact with an acid catalyst, Amberlyst resin [12]. The conversion process is comprised of four steps [19]: adsorption of the paraldehyde molecule at the catalyst, ring opening of the molecule to produce acetaldehyde, decomposition of unstable side reactants, and desorption of the acetaldehyde from the catalyst site. Kawasaki et al. [13] performed an experimental investigation of the process and reported the paraldehyde ringopening process to be the rate-determining step. Within the specified operating temperature range of $305 \mathrm{~K}$ to $322 \mathrm{~K}$, the reaction is promoted by the vaporization of acetaldehyde, which is also endothermic. The endothermic heat of reaction is calculated as the sum of the specific enthalpy change in the paraldehyde conversion and the latent heat of acetaldehyde vaporization. From the thermodynamic properties listed in Table 1 , this heat of reaction is calculated to be $189.5 \mathrm{~kJ} / \mathrm{kmol}[20]$.

The equilibrium constant of the reaction is a function of temperature and is written as:

$$
\ln K(T)=-\frac{1}{R T}\left(\Delta h_{298}-T \Delta s_{298}\right)
$$

The changes in specific enthalpy and specific entropy may be reasonably assumed to be constant across the small temperature range considered. The equilibrium constant also relates the mole fractions of paraldehyde and acetaldehyde according to [4]: 


$$
K(T)=\frac{a_{\mathrm{A}}^{3} y_{\mathrm{A}}^{3}}{a_{\mathrm{Pa}} y_{\mathrm{Pa}}}
$$

Assuming an ideal reaction, the activity coefficients ( $a_{\mathrm{A}}$ and $\left.a_{\mathrm{Pa}}\right)$ take values of unity; thus the equilibrium mole fractions of the chemical species are dependent on temperature alone. With known mole fractions and temperature, the equilibrium pressure of the mixture is:

$$
P_{\mathrm{eq}}=y_{\mathrm{A}} P_{\mathrm{A}, \mathrm{sat}}(T)+y_{\mathrm{Pa}} P_{\mathrm{Pa}, \mathrm{sat}}(T)
$$

The saturation pressures for acetaldehyde and paraldehyde are taken from [21, 22].

\subsubsection{Thermodynamic analysis}

Equilibrium pressure is calculated by combining Eqs. 1 to 3 . The results are plotted in Figure 2 as a function of acetaldehyde mole fraction for the chosen cold and hot reactor temperatures $(305 \mathrm{~K}$ and $322 \mathrm{~K}$ ). The two symbols in the graph mark the equilibrium mole fractions corresponding to the two reactor temperatures, of 0.117 and 0.239 , respectively. In accordance with the endothermic nature of the acetaldehyde production, the equilibrium mole fraction of acetaldehyde increases with temperature. However, this trend also implies that if the mixture leaves the endothermic reactor at equilibrium composition, the (hotter) exothermic reactor would produce more acetaldehyde (instead of regenerating paraldehyde) to achieve the new equilibrium state. Without the paraldehyde regeneration, the heat pump no longer has an exothermic process to release heat and the system loop becomes unsustainable.

To achieve a sustainable chemical heat pump with the proposed circuit, the mixture in the endothermic reactor must exceed the thermodynamic equilibrium state in order to induce the reverse reaction in the high-temperature exothermic reactor. One viable method for exceeding the equilibrium mole fraction is by pervaporation, a process (discussed in Section 3) in which the reaction products are separated via a selective membrane to artificially shift the mixture away from equilibrium and enable extended reaction. 


\subsubsection{Chemical reaction analysis}

Along with the equilibrium mole fraction limitations of the chemical species, reaction rates must also be considered to size appropriate reactor volumes in the heat pump design. Kawasaki et al. [14] empirically derived the following reaction rate for paraldehyde depolymerization as a function of temperature and paraldehyde concentration:

$$
\frac{r_{\mathrm{Pa}}}{\rho}=\left(\frac{2.92 \times 10^{22} \cdot C_{\mathrm{Pa}}}{1-0.132 C_{\mathrm{Pa}}}\right) \exp \left(\frac{-1.80 \times 10^{4}}{T}\right)
$$

A reaction rate model for the reverse process (polymerization of acetaldehyde) is not reported in the literature, and thus is not considered here. For the depolymerization model, the mass concentration of paraldehyde can be related to the acetaldehyde mole fraction as:

$$
C_{\mathrm{Pa}}=\frac{1-y_{\mathrm{A}}}{\left(1-y_{\mathrm{A}}\right) M_{\mathrm{Pa}}+y_{\mathrm{A}} M_{\mathrm{A}}}
$$

The mixture density is a function of component densities and the mass fraction of acetaldehyde:

$$
\rho=\frac{\rho_{\mathrm{Pa}} \cdot \rho_{\mathrm{A}}}{\left(\rho_{\mathrm{Pa}}-\rho_{\mathrm{A}}\right) \cdot x_{\mathrm{A}}+\rho_{\mathrm{A}}}
$$

In the selected temperature range, the densities of paraldehyde and acetaldehyde were assumed constant at $994 \mathrm{~kg} / \mathrm{m}^{3}$ and $1.52 \mathrm{~kg} / \mathrm{m}^{3}[21,22]$.

With the reaction rate known, the requisite volume for the endothermic plug flow reactor can be determined with:

$$
V=F \cdot \int_{0}^{y} \frac{d X}{-r}
$$

The molar flow rate $(F)$ in Eq. 7 refers to the chemical reactant (paraldehyde in the endothermic reactor, acetaldehyde in the exothermic reactor) and not the total mixture. The integral is solved 
through a change of variables by relating the chemical conversion of paraldehyde to the change in acetaldehyde mole fraction defined as:

$$
X_{\mathrm{Pa}}=\frac{y_{\mathrm{A}, 2}-y_{\mathrm{A}, 1}}{3-2 y_{\mathrm{A}, 2}}
$$

In addition, the conversion of acetaldehyde in the exothermic reactor can be related to the mole fractions as:

$$
X_{\mathrm{A}}=\frac{3\left(y_{\mathrm{A}, 3}-y_{\mathrm{A}, 4}\right)}{3-2 y_{\mathrm{A}, 4}}
$$

Since no reaction occurs across the work input device or the expansion valve, $y_{\mathrm{A}, 3}=y_{\mathrm{A}, 2}$ and $y_{\mathrm{A}, 4}$ $=y_{\mathrm{A}, 1}$.

With known molar flow rates and reactant conversions, the heat transfer in each reactor is defined as:

$$
Q=F \cdot X \cdot \Delta_{\mathrm{r}} h_{\mathrm{T}}
$$

The theoretical isentropic work input is then equal to the difference between the reactor heat transfer rates. As the required work is defined by the volumetric flow rate (constant due to the assumption of fixed fluid densities) multiplied by the pressure difference across the reactors, the efficiency of the work input can be defined as:

$$
\eta_{\mathrm{w}}=\frac{Q_{\mathrm{exo}}-Q_{\mathrm{end}}}{\dot{V} \cdot \Delta P}
$$

The system coefficient of performance (COP) is the ratio of endothermic transport to the required work input. Due to the small difference between the defined reservoir temperatures in the current work, the Carnot COP achieves a large value of 17.94. The ratio of the COP to the Carnot COP defines the exergy (or second-law) efficiency of the system.

\subsection{2-Propanol Dehydrogenation}


The performance of a comparable heat pump, based on the dehydrogenation of 2propanol reaction, is now evaluated. Use of 2-proponal dehydrogenation for a chemical heat pump was first proposed by Prevost and Bugarel in 1980 [15]. In the endothermic reactor, 2propanol dehydrogenates to acetone $\left(\left(\mathrm{CH}_{3}\right)_{2} \mathrm{CO}\right)$ and diatomic hydrogen. The acetone and hydrogen combine in the reverse, exothermic reaction to regenerate the 2-propanol. At atmospheric pressure, the optimum reaction temperatures are approximately $350 \mathrm{~K}$ for the dehydrogenation and $470 \mathrm{~K}$ for the reverse reaction [16]. As with the paraldehyde system, the endothermic reaction is not spontaneous and must be driven by catalysis. With the presence of hydrogen as a supercritical fluid, the system can be operated at constant pressure (neglecting frictional losses), eliminating the need for mechanical work input.

Saito et al. [17] proposed a waste heat recovery design concept with the 2-propanol reaction driven by a distillation column at fixed pressure. Waste heat is supplied to the endothermic reactor and the reboiler of the distillation column. The product mixture is fed into the lower section of the distillation column where the vaporized acetone and hydrogen gas rise to the top. This mixture is removed from the column, cooled by heat rejection to the surroundings, and fed into the exothermic reactor. The exothermic product is then returned to the column to separate the liquid 2-propanol generated. To make up for heat rejection to the surroundings and to achieve optimal exothermic reaction temperatures, a heat exchanger is installed between the distillation column and the exothermic reactor. As the column achieves phase separation of the reactants and products, parasitic mass in both reactors is minimized. Unlike the paraldehyde system, the reactors in the 2-proponal system are not adversely affected by the upstream conditions and do not require a pervaporation process.

This distillation column design for the 2-propanol dehydrogenation reaction is not a viable option for the defined thermal reservoirs $(305 \mathrm{~K}$ and $322 \mathrm{~K}$ ) chosen for this work due to 
the excess difference in temperature of the typical reactions. Given the boiling point of acetone of $330 \mathrm{~K}$ at atmospheric pressure, the distillation column would not sustain the system loop as the liquid acetone could not be transported through the distillation column to the exothermic reactor. Thus the current investigation retains the four-component design in Fig. 1 for the 2propanol reaction. Even though this reversible reaction preferentially occurs within a higher temperature range, it can serve to bring about thermal upgrading provided equilibrium mole fraction limitations can be exceeded to the desired value (mostly through membrane separation). This comparison is focused on the reactor size requirement associated with the desired value of heat load.

In the present case, the endothermic reaction is dehydrogenation of 2-propanol (IPA), taking place at 350-360 K when in contact with Raney Nickel as a catalyst, and the exothermic reaction is hydrogenation of acetone (Ac), occurring at 420-480 K [18]. The reaction equations are shown as follows:

$$
\begin{aligned}
& \left(\mathrm{CH}_{3}\right)_{2} \mathrm{CHOH}_{(\mathrm{l})} \rightarrow\left(\mathrm{CH}_{3}\right)_{2} \mathrm{CO}_{(\mathrm{g})}+\mathrm{H}_{2(\mathrm{~g})} \\
& \Delta h=100.4 \mathrm{~kJ} / \mathrm{mol} \\
& \left(\mathrm{CH}_{3}\right)_{2} \mathrm{CO}_{(\mathrm{g})}+\mathrm{H}_{2(\mathrm{~g})} \rightarrow\left(\mathrm{CH}_{3}\right)_{2} \mathrm{CHOH}_{(\mathrm{g})} \\
& \Delta h=-55.0 \mathrm{~kJ} / \mathrm{mol}
\end{aligned}
$$

At any given temperature, the partial pressures tend toward an equilibrium characterized by the equilibrium constant of the reaction defined as:

$$
\ln K=\ln P_{\mathrm{Ac}}+\ln P_{\mathrm{H} 2}-\ln P_{\mathrm{IPA}}
$$

Applying the thermodynamic properties listed in Table 2, the equilibrium constant can be calculated as a function of temperature using:

$$
\ln K=\ln K_{298}+\int_{298}^{T} \frac{\Delta_{\mathrm{r}} h_{\mathrm{T}}^{0}}{R T^{2}} d T
$$


The partial pressures also relate the number of moles of the different species to the extent of 2propanol conversion. Indeed, starting from an initial mixture of $n_{\mathrm{IPA}, 0}+n_{\mathrm{Ac}, 0}+n_{\mathrm{H} 2,0}$ moles, and assuming a conversion equal to $X_{\mathrm{IPA}}$, the composition at time $t>0$ is as follows:

- $\quad n_{\mathrm{IPA}, 0^{*}}\left(1-X_{\mathrm{IPA}}\right)$ moles of 2-propanol,

- $n_{\mathrm{Ac}, 0}+n_{\mathrm{IPA}, 0} \cdot X_{\mathrm{IPA}}$ moles of acetone,

- $n_{\mathrm{H} 2,0}+n_{\mathrm{IPA}, 0} \cdot X_{\mathrm{IPA}}$ moles of hydrogen, and

- $\quad$ total number of moles $n_{\mathrm{TOT}}=n_{\mathrm{IPA}, 0} \cdot\left(1+X_{\mathrm{IPA}}\right)+n_{\mathrm{Ac}, 0}+n_{\mathrm{H} 2,0}$.

The partial pressure of each species $\left(P_{\mathrm{i}}\right)$ is finally obtained by multiplying the total pressure $P_{\mathrm{T}}$ by the ratio of $n_{\mathrm{i}}$ and $n_{\mathrm{TOT}}$ :

$$
P_{\mathrm{i}}=P_{\mathrm{T}} \frac{\theta_{\mathrm{i}}+\varepsilon_{\mathrm{i}} X_{\mathrm{IPA}}}{1+X_{\mathrm{IPA}}+\theta_{\mathrm{Ac}}+\theta_{\mathrm{H} 2}}
$$

with $\theta_{\mathrm{i}}=\mathrm{n}_{\mathrm{i}, 0} / \mathrm{n}_{\mathrm{IPA}, 0}, \varepsilon_{\mathrm{IPA}}=-1$ and $\varepsilon_{\mathrm{Ac}}=\varepsilon_{\mathrm{H} 2}=1$.

\subsubsection{Thermodynamic analysis}

Combining Eq. 13A and Eq. 14, the logarithm of the equilibrium constant $K$ is plotted in Figure 3 as a function of 2-propanol conversion for $\theta_{\mathrm{Ac}}=\theta_{\mathrm{H} 2}=0$ (neither acetone nor hydrogen is present in the initial mixture), and for a total pressure $P_{\mathrm{TOT}}=10^{5} \mathrm{~Pa}$. Using Eq. $13 \mathrm{~B}$, the values of $K$ corresponding to the selected reactor temperatures $(305 \mathrm{~K}$ and $322 \mathrm{~K}$ ) can be calculated, and Fig. 3 can be used to evaluate the conversion at equilibrium for both temperatures: we obtain $2 \%$ and $4 \%$ conversion, respectively, in the two reactors. This result is in accordance with the endothermic nature of the 2-propanol dehydrogenation, with the chemical conversion (i.e., the percentage of reactants that are converted to products) increasing with temperature according to the Le Chatelier principle. It also means that if the mixture exiting the endothermic reactor is at equilibrium composition, the new equilibrium in the exothermic reactor 
(that corresponds to a lower partial pressure of 2-propanol) will be reached by producing more acetone and hydrogen: the system loop would thus be unsustainable.

One way to achieve a sustainable loop with this reversible reaction is to increase the chemical conversion of 2-propanol in the endothermic reactor beyond the equilibrium value. This can be done by removing at least one of the products during the reaction. Indeed, since the reaction is reversible, a decrease in the concentration of products has the effect of shifting the equilibrium to decrease the concentration of the reactants. Thus, removing hydrogen during reaction increases the 2-propanol conversion, and partial pressure of 2-propanol at the exit of the endothermic reactor is decreased below the equilibrium partial pressure calculated at the temperature of the exothermic reactor. Removal of hydrogen can be performed using the pervaporation process discussed below in Section 3.

\subsubsection{Chemical reaction analysis}

As with the paraldehyde/acetaldehyde system, reaction rates must be considered along with equilibrium limitations to size reactor volumes. Rioux and Vannice [23] derived the following reaction rate for 2-propanol dehydrogenation with copper catalyst as a function of partial pressures and temperature, for temperatures above $425 \mathrm{~K}$ :

$$
-\frac{1}{r_{\mathrm{IPA}}}=\frac{1}{r_{\mathrm{Ac}}}=\frac{\left(1+K_{\mathrm{IPA}} P_{\mathrm{IPA}}+K_{\mathrm{H}}^{1 / 2} P_{\mathrm{H}}^{1 / 2}+K_{\mathrm{Ac}} P_{\mathrm{Ac}}+K^{\prime} P_{\mathrm{Ac}} P_{\mathrm{H}}^{1 / 2}\right)^{2}}{k K_{\mathrm{IPA}} P_{\mathrm{IPA}}}
$$

In this equation, $K_{\mathrm{IPA}}, K_{\mathrm{Ac}}$ and $K^{\prime}$ are temperature-dependent equilibrium constants, and $k$ is the rate constant that also depends on temperature.

With the reaction rate known, and assuming that Eq. 15 is valid in the $300-400 \mathrm{~K}$ temperature range, the required volume for the endothermic plug flow reactor can be determined according to Eq. 7, where the molar flow rate is that of 2-propanol. Thus the value of the 2propanol flow rate must be as small as possible to yield small volumes: it is fixed at the 
minimum value that still reaches the desired heat load. Now heat transfer in both reactors is given by Eq. 10. If we plot the variations of $Q$ with $F_{\text {IPA }}$ for different values of 2-propanol conversion (Figure 4), it appears that a molar flow rate of $0.003 \mathrm{~mol} / \mathrm{s}$ is sufficient to ensure a 5 W heat load in the endothermic reactor.

Indicators of system performance are the coefficient of performance $(\mathrm{COP})$, the percent of upgraded heat, and the exergy efficiency. The COP is calculated from the ratio of endothermic transport rate to the required work input; the work input, $W_{\mathrm{in}}$, is the work supplied to transport the reaction products from one reactor to the other:

$$
C O P=\frac{Q_{\text {end }}}{W_{\text {in }}}
$$

The second indicator, namely the percent of upgraded heat (denoted $\% Q$ ), is the ratio of the heat released, $Q_{\text {exo }}$, from the exothermic reactor to that stored in the endothermic reactor, $Q_{\text {endo. }}$ The percent of upgraded heat is:

$$
\% Q=\frac{Q_{\text {exo }}}{Q_{\text {end }}}
$$

Finally, the exergy efficiency is the ratio of the exergy output, $\Phi_{\text {out }}$, to the exergy input, $\Phi_{\text {in }}$. With $T_{0}$ being the inlet temperature of the reactor, the exergy input is calculated as:

$$
\Phi_{\text {in }}=Q_{\text {end }}\left(1-\frac{T_{0}}{T_{\text {end }}}\right)+W_{\text {in }}
$$

where the first term is the thermal exergy and the second term the work supplied to transport the reaction products. The exergy output is calculated from:

$$
\Phi_{\text {out }}=Q_{\text {exo }}\left(1-\frac{T_{0}}{T_{\text {exo }}}\right)
$$

The exergy efficiency is then: 


$$
\eta_{2}=\frac{\Phi_{\text {out }}}{\Phi_{\text {in }}}
$$

\section{Proposed Reactor Design}

\subsection{Pervaporation Theory}

Equilibrium constraints pose a design challenge for many industrial chemical processes, notably in organic processes involving hydrogen such as the production of synthesis gas ( $\mathrm{CO}$ and $\mathrm{H}_{2}$ ) or methanol $[24,25]$. To improve production yields with pervaporation processes, membrane reactors have been widely studied through modeling efforts [26, 27]. Similar to traditional reactors, these novel systems operate in either continuously stirred tank reactor (CSTR) or plug flow reactor (PFR) modes.

For a membrane-modified PFR, the initial mixture enters the tube side of a concentric tube assembly while an inert gas (often referred as sweep gas) flows through the shell side. The tube itself is composed of the selective membrane along with a deposited catalyst to induce the desired reaction on the interior. For organic processes, membranes are typically palladium-based materials with known hydrogen permeability. The inert gas in annulus induces a partial pressure gradient across the membrane that creates the driving force for separation.

The performance of the membrane with respect to extending the reversible reaction to extend beyond its equilibrium state is dependent on multiple design factors including the ratio of sweep gas to reactant flow rates, reaction stoichiometry, membrane surface area, membrane selectivity, residence time, and pressure gradient across the membrane [24-27]. The exit composition is independent of permeation rate, which is fixed by the membrane thickness. The appropriate thickness is optimized between requisite strength and volume constraints on the

concentric PFR. While no viable membrane material was identified for the separation of 
paraldehyde and acetaldehyde, it is assumed that a suitable membrane exists for the remainder of this analysis. However, membrane materials with defined and selective hydrogen permeability are well known and viable for the 2-propanol system.

\subsection{Concentric Tube Reactor Concept}

While industrial applications and research studies have proven that reactive mixtures can exceed thermodynamic equilibrium concentrations with pervaporation, the process has been optimized only for open systems where undesirable products of reaction can be readily removed from the system. Thus, a concentration gradient can be maintained across the membrane, which induces further product separation and prolongs the reactant conversion. As the proposed chemical heat pump uses a closed-loop design with a fixed total mass, this means of affecting the equilibrium does not apply. The concentration gradient across the membrane would therefore eventually converge to zero and the reaction would again be limited by equilibrium being reached. Instead, a pressure gradient must be induced across the membrane to facilitate mixture separation. A nozzle-diffuser is proposed for the shell side of the membrane-modified PFR to generate this pressure gradient as a result of the Venturi effect (Figure 5).

Referring to the paraldehyde system, the inlet mixture to the endothermic reactor enters both the shell and tube sides, with Amberlyst resin deposited on the tube side of the membrane. While the inner flow undergoes depolymerization, the outer flow enters the nozzle-diffuser constriction to convert potential energy to kinetic energy. This creates a pressure drop and thus a radial concentration gradient across the tube membrane. In effect, the external non-reacting mixture acts as a sweep gas relative to the internal reacting mixture which remains below equilibrium to continue depolymerization as needed. 


\section{Results and Discussion}

\subsection{Paraldehyde Depolymerization}

A thermodynamic and kinetic analysis of the proposed chemical heat pump design is conducted using a MATLAB [28] code developed under the following assumptions:

- Ideal chemical reactions

- Isobaric reactors

- Perfect membrane selectivity

- Negligible friction losses

To satisfy the isobaric condition in both reactors, the mixture must undergo some temperature change during the reaction process. At constant pressure, production of acetaldehyde generates a temperature decrease as the endothermic process consumes heat from the surroundings. Thus flow in the endothermic reactor cools as the reaction progresses while flow in the exothermic reactor increases in temperature. This phenomenon is counter-intuitive with respect to traditional heat exchangers where the hot- and cold-side temperature difference diminishes along the length of the heat exchanger. Unlike a traditional heat exchanger, convection contributions in the chemical heat pump are overwhelmed by the reaction heat transfer and are assumed negligible. In any case, it is recommended that the endothermic reactor be operated colder than the low temperature reservoir and the exothermic reactor be operated warmer than the high temperature reservoir to prevent parasitic heat transfer.

The performance of the simulated chemical heat pump is computed according to the flow chart shown in Figure 6. The temperatures at both reactor inlets $\left(T_{1}\right.$ and $\left.T_{3}\right)$ are determined by a selected pinch-point temperature difference (the minimum temperature difference between the reservoir and the fluid in the reactor) of $0.5 \mathrm{~K}$. For the stated reservoir temperatures of $305 \mathrm{~K}$ 
and $322 \mathrm{~K}$, the inlet temperatures to the endothermic and exothermic reactors are $304.5 \mathrm{~K}$ and 322.5 K, respectively. Since the saturation pressure data for acetaldehyde was not available above $325 \mathrm{~K}$ [21], the outlet temperature of the exothermic reactor is artificially bounded at $324.5 \mathrm{~K}$.

With fixed temperatures, the thermodynamic state points of the chemical heat pump are then calculated from user-defined heat load in the endothermic reactor $\left(\mathrm{Q}_{\mathrm{end}}\right)$ and the acetaldehyde mole fraction at the reactor inlet $\left(\mathrm{y}_{\mathrm{A} 1}\right)$. Due to the lack of reaction across the expansion valve, the mole fraction exiting the exothermic reactor $\left(\mathrm{y}_{\mathrm{A} 4}\right)$ is equal to this user input. The pressures associated with the exothermic reactor $\left(\mathrm{P}_{3}\right.$ and $\left.\mathrm{P}_{4}\right)$ are obtained from Eq. 3 . The remaining mole fractions ( $\mathrm{y}_{\mathrm{A} 3}$ and $\mathrm{y}_{\mathrm{A} 4}$ ) are then solved for the reaction extents in the endothermic $\left(\mathrm{X}_{\mathrm{Pa}}\right)$ and exothermic $\left(\mathrm{X}_{\mathrm{A}}\right)$ reactor. The required molar flow rate of paraldehyde at the reactor inlet $\left(\mathrm{F}_{\mathrm{Pa}, 1}\right)$ is determined from Eq. 10. A mass balance then yields the molar flow rate of acetaldehyde at the exothermic reactor inlet. Eq. 10 is again solved for the heat transfer rate in the exothermic reactor $\left(\mathrm{Q}_{\mathrm{exo}}\right)$. The isentropic work input is obtained as the difference in heat transfer rates, while the actual work input is calculated using Eq. 11. With the work input known, the COP and exergy efficiency of the chemical heat pump can be calculated. The required size of the endothermic reactor is solved by numerical integration of Eq. 7 .

This design algorithm was implemented in the user-generated code and applied for various endothermic heat loads and endothermic inlet mole fractions of acetaldehyde to assess the performance of the chemical heat pump loop. Table 3 lists the results of this computation for various inlet mole fractions at the fixed endothermic heat input rate of $5 \mathrm{~W}$. For an acetaldehyde mole fraction of 0.3 , the reactor volume is minimized to $93 \mathrm{~cm}^{3}$ and the exergy efficiency maximized to 0.132. The temperature vs. mole fraction and pressure vs. specific volume diagrams at this mole fraction are plotted in Figsure 7 and 8. Mass balance of the coupled 
reactors prohibited physical solutions below this mole fraction for additional gains. As such, this entering acetaldehyde is a significant parasitic mass in the endothermic reactor as it displaces volume from the reacting paraldehyde. Such unwanted mass may be minimized through the addition of purification lines in parallel to both reactors, such that the exiting reactant is diverted back to the reactor inlet to lower the mole fraction of entering product, reducing the required reaction volume. However, this additional complexity may ultimately increase system volume from additional check valves and secondary pumps to direct the ancillary flow paths. The simulations also reveal that the endothermic heat load does not affect the state points of the loop, but only scales the mass flow and energy transfer rate as well as the endothermic reactor volume.

As the required inlet mole fractions of acetaldehyde at the endothermic reactor inlet already exceed the equilibrium condition, a separation process is necessary prior to catalysis to promote the depolymerization reaction. To achieve this, a second nozzle-diffuser concentric tube assembly may be installed upstream of the endothermic reactor (without a deposited catalyst). The constriction drives separation of the excess acetaldehyde through the membrane until the mole fraction of acetaldehyde is below the equilibrium value, at which time the forward reaction can be achieved in the endothermic reactor. An alternative solution is to extend the length of the endothermic reactor, with catalyst deposited only in the downstream section of the membrane wall.

As seen from the results in Table 3, increasing the inlet mole fraction of acetaldehyde reduces the system $\mathrm{COP}$ and increases the required endothermic reactor volume (not including the separation process prior to reaction). In addition, the required minimum efficiency of the work input is also reduced, expanding the choice of suitable devices that may be selected. Thus under the stated assumptions, the system COP is related to the work input efficiency according to the following correlating equation: 


$$
C O P=0.289 \eta_{w}^{2}+3.091 \eta_{w}-0.463
$$

With this relationship, the performance of the present system is ascertained from the efficiency of the required work input. While the model predicts large COP values for high-efficiency work input, it should be reiterated that the Carnot COP for the system is inherently large due to the small difference between the reservoir temperatures. As a result, the exergy efficiency remains less than 0.15 even with work input at high-efficiency.

As noted in the literature [12], sub-atmospheric pressures are needed to operate the chemical heat pump at the specified temperatures (leading to hermetic sealing in a real system). It was shown above that the defined endothermic heat load does not affect the state points of the loop but does scale the reactor size. Varying the endothermic heat load from 0.1 to $100 \mathrm{~W}$, the required reaction volume (with 0.3 mole fraction of acetaldehyde at the inlet) increased from 2 to $2,000 \mathrm{~cm}^{3}$. This volume accounts for the space needed to achieve the desired paraldehyde conversion, but not the additional volume required to separate excess acetaldehyde from the inlet mixture or the volume of the Amberlyst resin.

\subsection{2-Propanol Dehydrogenation}

A similar analysis as that for the paraldehyde/acetaldehyde $(\mathrm{Pa} / \mathrm{A})$ chemical heat pump system above was also conducted for the 2-propanol/acetone/hydrogen (IPA/Ac/H) system. The same assumptions of ideal chemical reactions, isobaric reactors, perfect membrane selectivity and negligible friction losses were again made. Temperatures in the endothermic and exothermic reactors were also fixed as before at $305 \mathrm{~K}$ and $322 \mathrm{~K}$, respectively, and the total pressure in both reactors was fixed at $10^{5} \mathrm{~Pa}$. The heat load $Q$ (i.e., the energy stored in the chemical bonds of the reaction products) was also fixed at $5 \mathrm{~W}$. With these chosen parameters, the chemical heat pump operation can be solved entirely for a given chemical conversion $X_{\mathrm{IPA}}$ and a selected initial 
composition at the endothermic reactor inlet. For each case, an equimolar initial mixture of acetone and hydrogen has been considered.

Partial pressures inside the reactor and the remaining unknown composition (endothermic outlet) are found using Eq. 14 and a mass balance. The required endothermic reactor volume is then solved by numerical integration of Eq. 7, knowing that the rate of reaction $r_{\mathrm{Ac}}$ is related to $X_{\mathrm{IPA}}$ by combining Eq. 14 and Eq. 15. As explained in Section 2.1.2, the molar flow rate $F_{\mathrm{IPA}}$ is determined from Eq. 10, for fixed values of $Q$ and $X_{\mathrm{IPA}}$ and at a given temperature.

Results are computed for different endothermic heat loads and inlet compositions to assess the possibility of storing a desired amount of power in chemical bonds at the endothermic reactor level under strict volume constraints. This power is then released in the exothermic reactor where the catalyzed reverse reaction occurs. Temperature and mass of the catalyst are important criteria for the process, but it was found that the parameter that most influences the system efficiency was the chemical conversion of 2-propanol. Table 4 lists solutions in terms of molar flow rate, volume, and composition at a constant endothermic heat load equal to $5 \mathrm{~W}$. Case 1 corresponds to the unsustainable loop, the case where equilibrium at the exothermic reactor level leads to the production of more acetaldehyde instead of regenerating paraldehyde.

As stated in Section 2.1, limitation of the endothermic reaction to equilibrium conditions must be overcome to achieve a sustainable loop. However, if the endothermic reaction is promoted so that the chemical conversion $X_{\text {IPA }}$ doubles to $4 \%$ from $2 \%$ (case 2), the corresponding reactor size is approximately $8000 \mathrm{~cm}^{3}$, even with a molar flow rate of 0.003 $\mathrm{mol} / \mathrm{s}$. For the same temperature and heat load conditions, the $\mathrm{Pa} / \mathrm{A}$ system required a volume of about $93 \mathrm{~cm}^{3}$ for the endothermic reactor. The larger volume requirement for the IPA reaction is attributed to the small values of $r_{\mathrm{Ac}}$, calculated from Eq. 15 and plotted in Figure 9 as a function of 2-propanol chemical conversion. Indeed, the reversible reaction of 2-propanol 
dehydrogenation usually takes place within a temperature range higher than $305 \mathrm{~K}$, which means that $r_{\mathrm{Ac}}$ is a maximum in the range of $350 \mathrm{~K}$ to $360 \mathrm{~K}$ and decreases with temperature due to the endothermic nature of the reaction.

In order to operate at a reasonable reactor size, it is important to increase 2-propanol conversion far beyond the equilibrium conversion $X_{\mathrm{IPA}}$ of $2 \%$. As shown in Table 4, allowing the temperature of the endothermic reactor to increase to $310 \mathrm{~K}$ (instead of $305 \mathrm{~K}$ ), a 2-propanol conversion of $65 \%$ reduces the reactor size to $60 \mathrm{~cm}^{3}$ for the given heat load and exothermic reactor temperature (case 3). The required reactor size further reduces to about $48 \mathrm{~cm}^{3}$ for $Q=4$ W under the same conditions. To achieve a conversion $X_{\text {IPA }}$ that is as high as possible (aside from a pervaporation approach), a separation process may be used prior to catalysis in order to promote the dehydrogenation reaction. Indeed, with a larger amount of reactants, the reaction rate would also increase. Thus, a better conversion is obtained by separating the unreacted acetone and hydrogen mixture from the 2-propanol that is produced, prior to the endothermic reactor inlet. It may be noted that the calculated volumes in Table 4 account only for the volume needed to achieve the desired conversion for a fixed $Q$, and not the additional volume required to separate the unreacted reactants from the products: there is a trade-off between increasing reaction rate through separation in order to decrease the reactor size, and the corresponding increase in total volume resulting from the introduction of the separation process.

The exergy efficiency associated with the system is related to the coefficient of performance (combining Eqs. 16 to 20) according to

$$
\eta_{2}=\frac{\operatorname{COP}\left(1-\frac{T_{\text {endo }}}{T_{\text {exo }}}\right)}{1+\frac{\operatorname{COP}}{\% Q}\left(1-\frac{T_{\text {exo }}}{T_{\text {endo }}}\right)}
$$


With this relationship, the designer can ascertain the performance of the entire system based on the COP, which is a function of the work input efficiency. For a COP of 2.38 (corresponding to case 1 in Table 3), and a $\% Q$ of approximately 0.4 , we obtain an exergy efficiency of 0.19 . This value is slightly greater than the efficiency obtained with the $\mathrm{Pa} / \mathrm{A}$ system (equal to 0.132 ) under similar conditions. The exergy efficiency as a function of the COP for this system is plotted in Figure 10 for different values of $\% Q$.

\subsection{Comparison with Other Technologies}

The main criteria for assessing technologies for electronics thermal management are the environmental impact of the cooling system, its energy efficiency, required volume, and complexity of the design. Candidate technologies for comparison with the CHP include heat pipes [29, 30], vapor compression and sorption cooling systems [5-9], and thermoelectric coolers [31]. A summary of a qualitative comparison is presented in Table 5. When comparing possible cooling technologies in this manner, no single system stands out as being ideal on all fronts.

Technologies that use a refrigerant fluid (heat pipes, vapor compression and sorption cooling systems) that can possibly leak into the atmosphere through seal degradation, repair, or accident pose environmental and/or health risks that render the use of such technologies less attractive. Heat pipes can passively and efficiently spread the heat load over a relatively largearea heat sink with the working fluid being transported by capillary forces developed in the internal wick structure. However, a limiting factor to their operation can be the capillary limit which is set by the pumping capacity of the wick structure and is a strong function of the operating orientation. In the case of a thermoelectric cooler, the maximum COP for a $\Delta \mathrm{T}$ of 20 $\mathrm{K}$ varies from 0.3 to 1.1 , depending on the semiconductor material that is used. In comparison, the chemical heat pump system, with a COP of 1.0 to 2.4 and no orientation-dependence, offers a promising alternative for energy efficiency in electronics cooling. 
However, very little space is allocated to the cooling solution in electronic systems, and the volume requirement may be the most constraining. Heat pipes and thermoelectric coolers are inherently compact but their performance can be affected by operating temperatures and power inputs that are outside design values. Chemical heat pumps not only need extra heat exchangers, but also large reactor areas for the chemical reactions to take place efficiently; the system would therefore benefit strongly from research aimed at size reduction. One approach toward size and weight reduction of a refrigerating loop (including vapor-compression) involves the use of an ejector [32] instead of a compressor for driving the liquid-vapor mixture from the endothermic to the exothermic reactor. The need for lubricating oil in the compressor is thereby eliminated. The ejector also has the advantage that the resulting COP for CHP and vapor-compression systems would be higher due to a lower work input.

Finally, the systems with the lowest complexity have no moving parts, no working fluid, and no dynamic seals. The thermoelectric cooler satisfies these requirements and can also be cost-competitive with other systems; its major drawback is its relatively low COP. Heat pipes also have low complexity and cost. Use of an ejector instead of a compressor for pumping of the liquid-vapor mixture would increase complexity of the CHP but also increase the associated COP, thus making the system more efficient and attractive practically. The components of the CHP are also inexpensive. The complexity of existing systems may be compared through an analysis of parts counts and tolerance requirements, but it is difficult to forecast evolving developments and improvements in the technologies. In terms of cost and energy efficiency through COP estimation, however, it appears that a CHP holds good potential for environmentally friendly application in electronics cooling, assuming reasonable volume constraints. 


\section{Conclusions}

A detailed thermodynamic and kinetic study of paraldehyde depolymerization and 2propanol dehydrogenation is performed to assess the suitability of a reaction-based chemical heat pump to thermal management applications in consumer electronics. With both reaction systems, the limits set by thermodynamic equilibrium must be overcome for a sustainable heat pump operation. A novel concentric membrane tube plug flow reactor is proposed to address this need, including a nozzle-diffuser constriction on the shell side to generate pervaporation in a closed system.

For the paraldehyde reaction, an inlet flow mixture of 0.3 mole fraction acetaldehyde minimizes the endothermic reactor volume to $93 \mathrm{~cm}^{3}$ and maximizes the exergy efficiency to 0.132. The versatility of the present model can be improved through an experimental study which includes characterization of acetaldehyde polymerization to enable accurate sizing of the exothermic reactor, determination of acetaldehyde saturation pressures at higher temperatures to remove the artificial temperature boundary at the exothermic reactor outlet, and determination of activity coefficients associated with the reactions to increase accuracy in the calculation of equilibrium mole fractions.

Regarding the 2-propanol system, pervaporation combined with a separation process can lead to a conversion large enough to store the $5 \mathrm{~W}$ heat load with a reduced endothermic reactor volume of 60 cubic centimeters, depending on the membrane efficiency. A kinetic analysis combined with a modeling of the pervaporation process must be performed to assess the practical feasibility of the system. Given the endothermic reactor size reported, cooling of larger scale systems such as data centers may be a more viable application due to the relaxed volume constraints. Data centers may carry different temperature reservoirs than portable electronic systems, however, and some adjustment in cycle performance would be expected. 
The chemical heat pump has advantages including low cost, overall efficiency and lack of moving parts. However, its volume requirements pose a distinct drawback that must be overcome for practical use in electronics cooling. Indeed, in both the chemical reaction systems considered in this work, low exergy efficiencies resulted from the minimization of endothermic reactor volume. As such, chemical heat pump cooling may ultimately be more appropriate in applications with less stringent volume constraints such as data centers. This relaxation includes lower energy transport rates to reduce the amount of reaction required, larger temperature ranges to achieve faster reaction rates, and greater allowable volumes to better accommodate necessary reaction and product separation volumes.

\section{Acknowledgement}

The authors acknowledge financial support for this work from Intel Corporation.

\section{References}

1. S. V. Garimella, Advances in mesoscale thermal management technologies for microelectronics, Microelectronics J. 37 (2006) 1165-1185.

2. R. C. Chu, R. E. Simons, M. J. Ellsworth, R. R. Schmidt, V. Cozzolino, Review of cooling technologies for computer products, IEEE Trans. Device and Materials Reliability 4 (2004) 568-585.

3. S. Trutassanawin, E. A. Groll, S. V. Garimella, L. Cremaschi, Experimantal investigation of a miniature-scale refrigeration system for electronics cooling, IEEE Trans. Components and Packing Tech. 29 (2006) 678-687.

4. M. J. Moran, H. N. Shapiro, Fundamentals of engineering thermodynamics. $5^{\text {th }}$ edition. John Wiley \& Sons, 2004. 
5. S. N. Mumah, S. S. Adefila, E. A. Arinze, Properties generation procedures for first and second law analyses of ammonia-water heat pump system, Energy Conv. Mgmt 35 (1994) 727-736.

6. Y. Gupta, L. Metchop, A. Frantzis, P. E. Phelan, Comparative analysis of thermally activated, environmentally friendly cooling systems, Energy Conv. Mgmt 49 (2008) 1091-1097.

7. A. Haywood, J. Sherbeck, P. Phelan, G. Varsamopoulos, S. K. S. Gupta, Thermodynamic feasibility of harvesting data center waste heat to drive an absorption chiller, Energy Conv. Mgmt 58 (2012) 26-34.

8. R. Z. Wang, J. Y. Wu, Y. X. Xu, W. Wang, Performance researches and improvements on heat regenerative adsorption refrigerator and heat pump, Energy Conv. Mgmt 42 (2001) 233-249.

9. L. X. Gong, R. Z. Wang, Z. Z. Xia, C. J. Chen, Design and performance prediction of a new generation adsorption chiller using composite adsorbent, Energy Conv. Mgmt 52 (2011) 2345-2350.

10. W. Wongsuwan, S. Kumar, P. Neveu, F. Meunier, A review of chemical heat pump technology and applications, App. Therm. Eng. 21 (2001) 1489-1519.

11. S. Fujimoto, E. Bilgen, H. Ogura, $\mathrm{CaO} / \mathrm{Ca}(\mathrm{OH})_{2}$ chemical heat pump system, Energy Conv. Mgmt 43 (2002) 947-960.

12. H. Kawasaki, T. Watanabe, A. Kanzawa, Proposal of a chemical heat pump with paraldehyde depolymerization for cooling system. App. Therm. Eng. 19 (1999) 134-143.

13. H. Kawasaki, A. Kanzawa, T. Watanabe, Characteristics of chemical heat pump through kinetic analysis of paraldehyde depolymerization. J. Chem. Eng. Japan 31 (1998) 374380. 
14. H. Kawasaki, T. Watanabe, A. Kanzawa, Studies on paraldehyde depolymerization for chemical heat pump. Proceedings of ISAECSRT; 1995.

15. M. Prevost, R. Bugarel, Chemical heat pumps: System isopropanol-acetone-hydrogen. Proceedings of the International Conference on Energy Storage; 1980, 95-110.

16. Y. Chung, B. Kim, Y. Yeo, H. Song, Optimal design of a chemical heat pump using the 2propanol/acetone/hydrogen system. Energy 22 (1997) 525-536.

17. Y. Saito, H. Kaneyama, K. Yoshido, Catalyst-assisted chemical heat pump with reaction couple of acetone hydrogenation/2-propanol dehydrogenation for upgrading low-level thermal energy: Proposal and evaluation. Int. J. Energy Res. 11 (1987) 549-558.

18. W. Kitikiatsophon, P. Piumsomboon, Dynamic simulation and control of an isopropanolacetone-hydrogen chemical heat pump. ScienceAsia 30 (2004) 135-147.

19. P. J. Chong, G. Curthoys, Microcatalytic study of the depolymerization of 2,4,6Trimethyl-1,3,5-trioxan (paraldehyde) over mordenite surfaces. J. Chem. Soc., Faraday Trans. 178 (1982) 53-60.

20. T. Daubert, R. Danner, H. Sibul, C. Stebbins, Physical and thermodynamic properties of pure chemicals. Taylor \& Francis, 1989.

21. Acetaldehyde property data, http://cameochemicals.noaa.gov/chris/AAD.pdf. Last accessed October 10, 2011.

22. Paraldehyde property data, http://cameochemicals.noaa.gov/chris/PDH.pdf. Last accessed October 10, 2011.

23. R. M. Rioux, M. A. Vannice, Hydrogenation/dehydrogenation reactions: isopropanol dehydrogenation over copper catalysts. J. Catalysis. 216 (2003) 362-376. 
24. G. Marigliano, G. Barbieri, E. Drioli, Equilibrium conversion for a Pd-based membrane reactor. Dependence on the temperature and pressure. Chem. Eng. Proc. 42 (2003) 231236.

25. M. Rahimpour, S. Ghader, Enhancement of CO conversion in a novel Pd-Ag membrane reactor for methanol synthesis. Chem. Eng. Proc. 43 (2004) 1181-1188.

26. J. Sousa, A. Mendes, Modeling a catalytic membrane reactor with plug flow pattern and a hypothetical equilibrium gas-phase reaction with $\Delta n \neq 0$. Catalysis Today. 104 (2005) 336-343.

27. S. Rezai, Y. Traa. Equilibrium shift in membrane reactors: a thermodynamic analysis of the dehydrogenative conversion of alkanes. J. Membrane Sci. 319 (2008) 279-285.

28. MATLAB Documentation, R2010a Users Guide, 2010.

29. J. A. Weibel, S. V. Garimella, M. T. North. Characterization of evaporation and boiling from sintered-powder wicks fed by capillary action. Int. J. Heat Mass Transfer 53 (2010) 4204-4215.

30. R. Ranjan, J. Y. Murthy, S. V. Garimella, U. Vadakkan. A numerical model for transport in flat heat pipes considering wick microstructure effects. Int. J. Heat Mass Transfer 54 (2011) 143-168.

31. D. Wee, Analysis of thermoelectric energy conversion efficiency with linear and nonlinear temperature dependence in material properties. Energy Conv. Mgmt. 52 (2011) 3383-3390.

32. G. Grazzini, A. Milazzo, D. Paganini, Design of an ejector cycle refrigeration system, Energy Conv. Mgmt 54 (2012) 38-46. 
Tables

Table 1. Thermodynamic properties of paraldehyde and acetaldehyde.

\begin{tabular}{cccc} 
Property & Units & Paraldehyde & Acetaldehyde \\
\hline$h_{\mathrm{fg}}$ & $\mathrm{kJ} / \mathrm{mol}$ & -687.99 & -192.57 \\
$s$ & $\mathrm{~kJ} / \mathrm{mol}-\mathrm{K}$ & 0.234 & 0.181 \\
$h_{\mathrm{v}}$ & $\mathrm{kJ} / \mathrm{mol}$ & 44.23 & 26.37 \\
\hline
\end{tabular}

Table 2. Thermodynamic properties for 2-propanol dehydrogenation.

\begin{tabular}{ccccc} 
Property & Units & IPA & Ac & $\mathrm{H}$ \\
\hline$h_{\mathrm{fg}}$ & $\mathrm{kJ} / \mathrm{mol}$ & -272.70 & -217.10 & 0 \\
$s$ & $\mathrm{~kJ} / \mathrm{mol}-\mathrm{K}$ & 0.31 & 0.295 & 0.13 \\
$h_{\mathrm{v}}$ & $\mathrm{kJ} / \mathrm{mol}$ & 44 & 32 & 0.9 \\
\hline
\end{tabular}

Table 3. Solutions to chemical heat pump model with endothermic heat load of $5 \mathrm{~W}$ for $\mathrm{Pa} / \mathrm{A}$ system.

\begin{tabular}{ccccccc} 
Parameter & Units & Case 1 & Case 2 & Case 3 & Case 4 & Case 5 \\
\hline$y_{\mathrm{A} 1}$ & - & 0.30 & 0.32 & 0.34 & 0.36 & 0.38 \\
$y_{\mathrm{A} 2}$ & - & 0.332 & 0.353 & 0.375 & 0.396 & 0.418 \\
$X_{\mathrm{Pa}}$ & - & 0.0135 & 0.0144 & 0.0153 & 0.0163 & 0.0173 \\
$X_{\mathrm{A}}$ & - & 0.0395 & 0.0420 & 0.0446 & 0.0474 & 0.0502 \\
$P_{\text {end }}$ & $\mathrm{kPa}$ & 69.8 & 72.1 & 74.5 & 76.9 & 79.3 \\
$P_{\text {exo }}$ & $\mathrm{kPa}$ & 134 & 139 & 144 & 148 & 153 \\
$\mathrm{COP}$ & - & 2.38 & 1.79 & 1.42 & 1.17 & 0.98 \\
$\eta_{2}$ & - & 0.132 & 0.100 & 0.079 & 0.065 & 0.055 \\
mass rate & $\mathrm{g} / \mathrm{s}$ & 0.295 & 0.280 & 0.267 & 0.254 & 0.243 \\
$\eta_{\mathrm{w}}$ & - & 0.851 & 0.685 & 0.579 & 0.504 & 0.449 \\
$V_{\text {end }}$ & $\mathrm{cm}^{3}$ & 93.0 & 111 & 132 & 156 & 184 \\
\hline
\end{tabular}


Table 4. Solutions to chemical heat pump model with endothermic heat load of $5 \mathrm{~W}$ for the IPA/Ac/H system.

\begin{tabular}{ccccc} 
Parameter & Units & Case 1 & Case 2 & Case 3 \\
\hline$T_{\text {endo }}$ & $\mathrm{K}$ & 305 & 305 & 310 \\
$F_{\mathrm{IPA}}$ & $\mathrm{mol} / \mathrm{s}$ & 0.003 & 0.003 & 0.0005 \\
$\theta_{\mathrm{IPA}, 1}$ & - & 1 & - & 0.97 \\
$\theta_{\mathrm{IPA}, 2}$ & - & 0.98 & - & 0.34 \\
$\theta_{\mathrm{Ac}, 1}=\theta_{\mathrm{H} 2,1}$ & - & 0.02 & - & 0.03 \\
$\theta_{\mathrm{Ac}, 2}=\theta_{\mathrm{H} 2,2}$ & - & 0.06 & - & 0.66 \\
$X_{\mathrm{IPA}}$ & - & 0.02 & 0.04 & 0.65 \\
$X_{\mathrm{Ac}}$ & - & 0.04 & 0.05 & 0.95 \\
$V$ & $\mathrm{~cm}^{3}$ & - & 8000 & 60 \\
\hline
\end{tabular}

Table 5. Comparison of different cooling technologies.

\begin{tabular}{lcccc} 
Technology & $\begin{array}{c}\text { Environmental/ } \\
\text { health impact }\end{array}$ & $\begin{array}{c}\text { Energy } \\
\text { efficiency }\end{array}$ & $\begin{array}{c}\text { Volume } \\
\text { constraint }\end{array}$ & $\begin{array}{c}\text { Complexity and } \\
\text { cost }\end{array}$ \\
\hline Heat pipe & High & Medium to High & Low & Low \\
Vapor compression & High & Medium & Medium & Medium \\
Sorption & High & Medium & Medium & Medium \\
Thermoelectric & Low & Low & Medium & Low \\
CHP & Low & High & High & Low to Medium \\
\hline
\end{tabular}




\section{Figure Captions}

Figure $1 \quad$ Idealized chemical heat pump cycle.

Figure 2 Equilibrium reactor pressure as a function of acetaldehyde mole fraction.

Figure 3 Equilibrium constant as a function of 2-propanol conversion.

Figure 4 Heat load vs. 2-propanol molar flow rate for different conversion values.

Figure 5 Concept of a pervaporation plug flow reactor for a chemical heat pump with membrane tube (reaction catalyst deposited on tube interior) and nozzle-diffuser constriction.

Figure 6 Paraldehyde chemical heat pump solution flow chart.

Figure 7 Temperature vs. mole fraction plot with 0.3 mole fraction of acetaldehyde at the endothermic reactor inlet.

Figure 8 Pressure vs. specific volume with 0.3 mole fraction of acetaldehyde at the endothermic reactor inlet.

Figure 9 Rate of reaction as a function of 2-propanol conversion.

Figure 10 Exergy efficiency as a function of COP for different amounts of upgraded heat. 


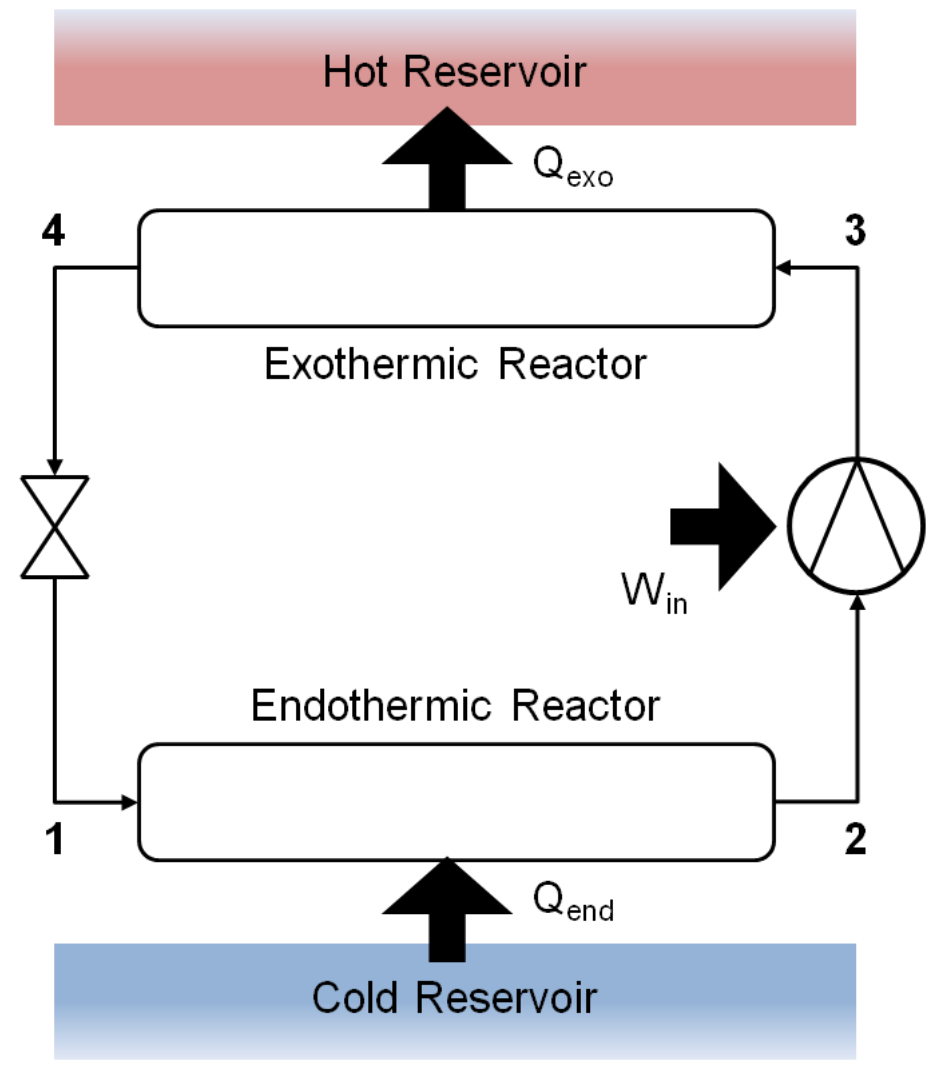

Figure 1: Idealized chemical heat pump cycle. 


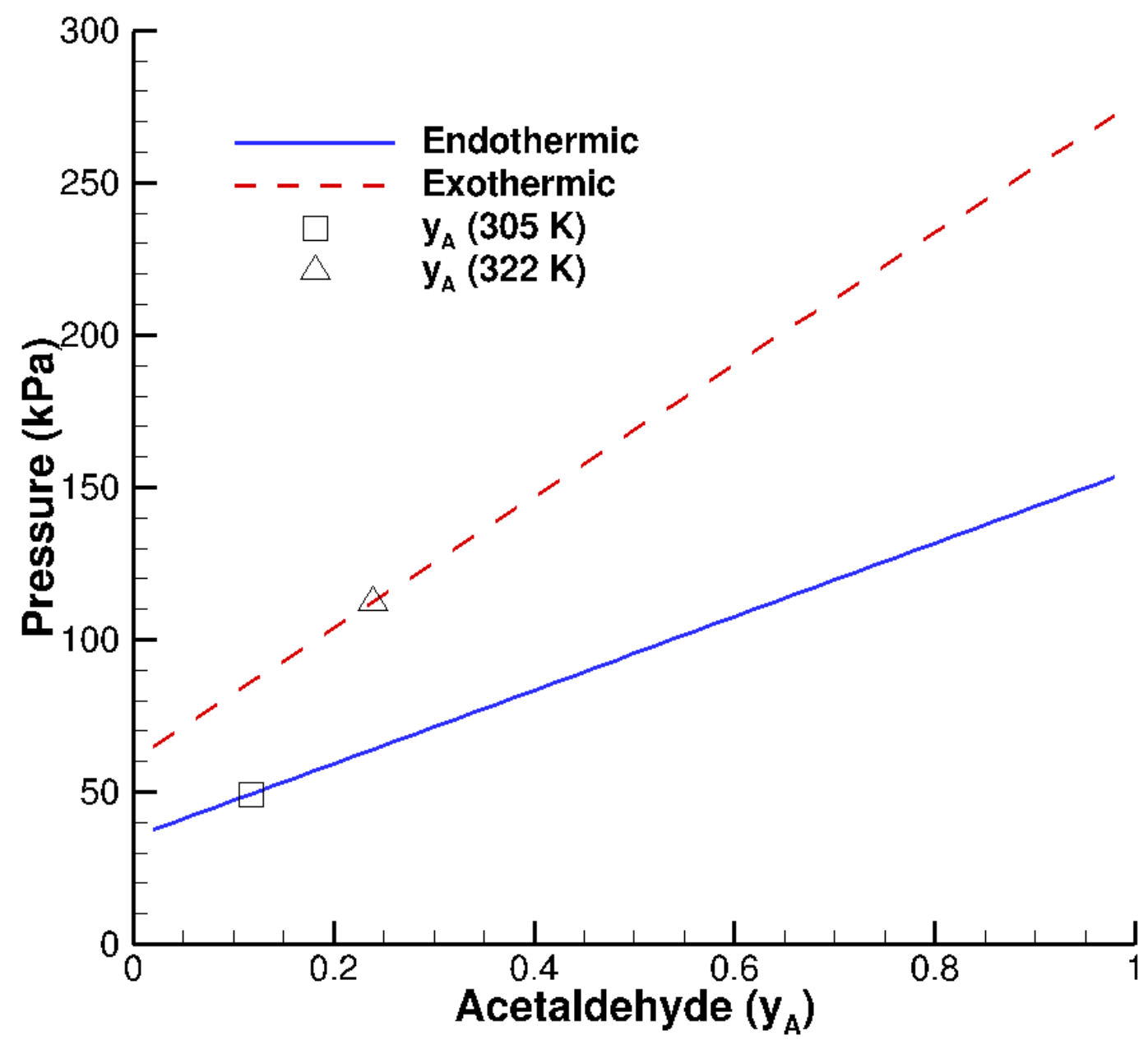

Figure 2: Equilibrium reactor pressure as a function of acetaldehyde mole fraction. 


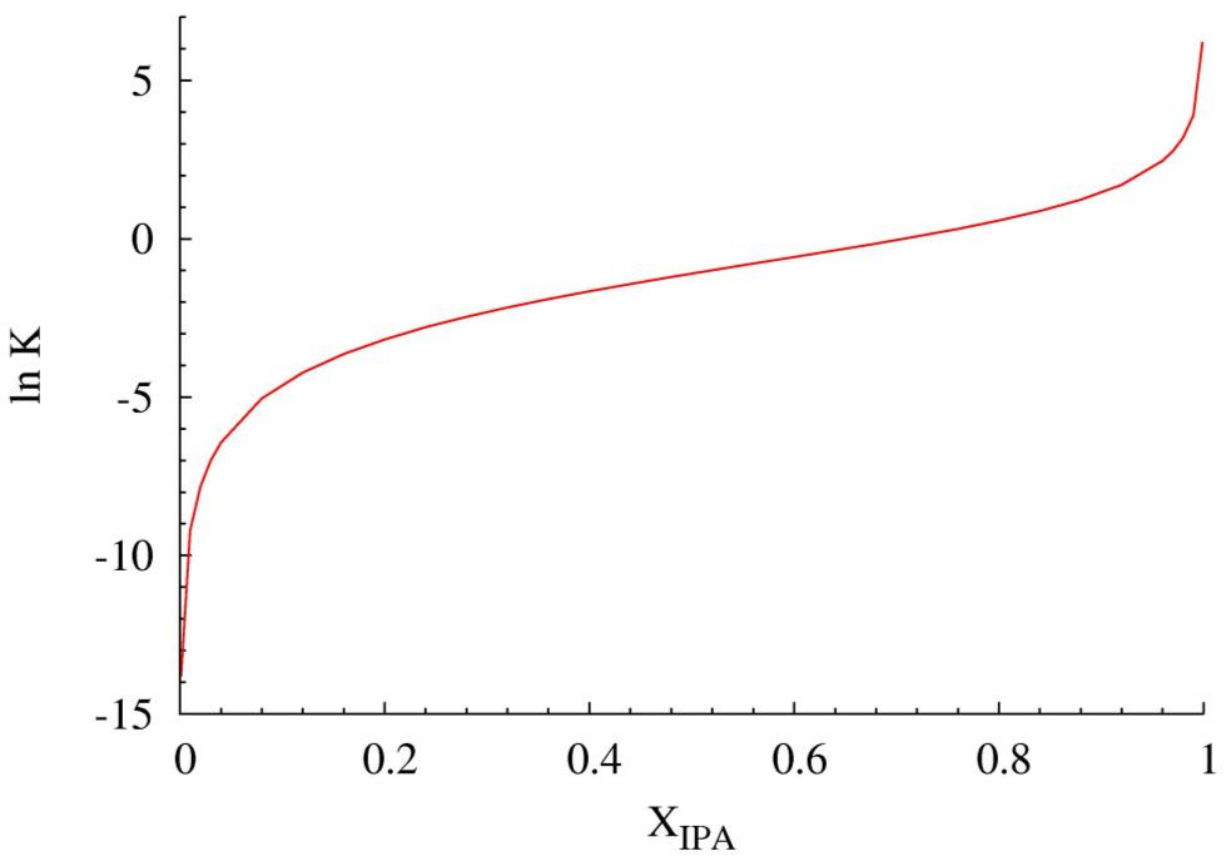

Figure 3: Equilibrium constant as a function of 2-propanol conversion. 


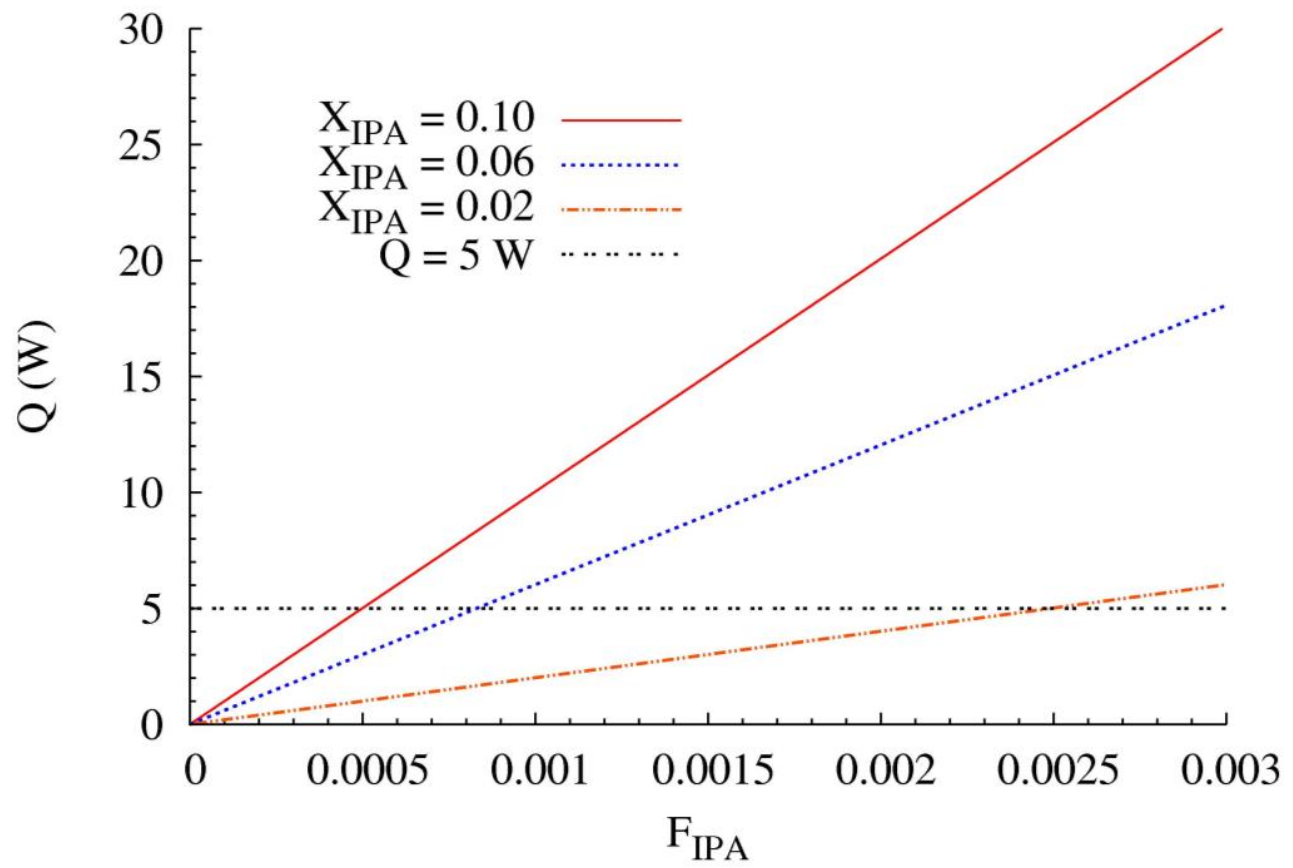

Figure 4: Heat load vs. 2-propanol molar flow rate for different conversion values. 


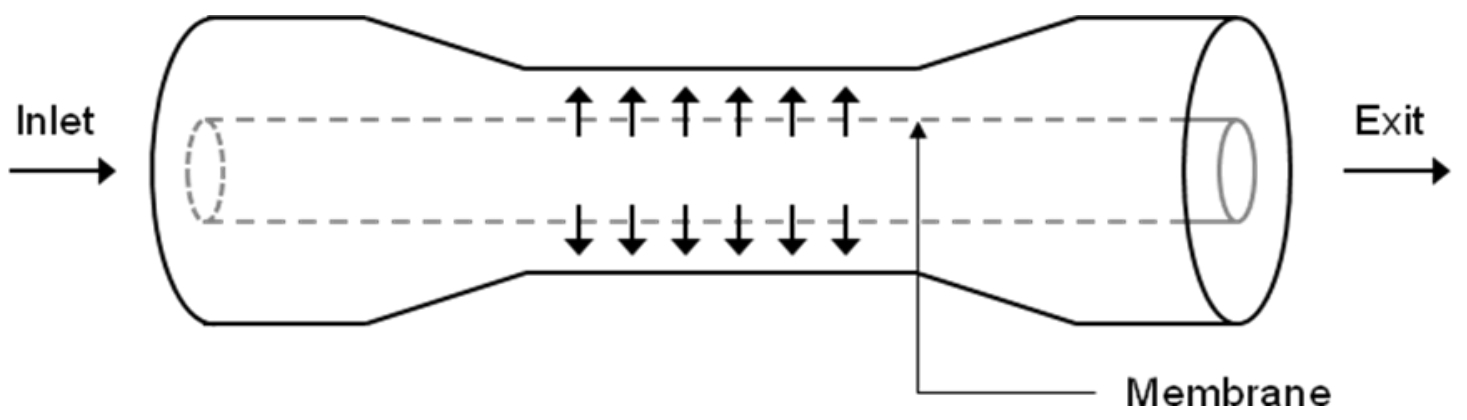

Figure 5: Concept of a pervaporation plug flow reactor for a chemical heat pump with membrane tube (reaction catalyst deposited on tube interior) and nozzle-diffuser constriction. 


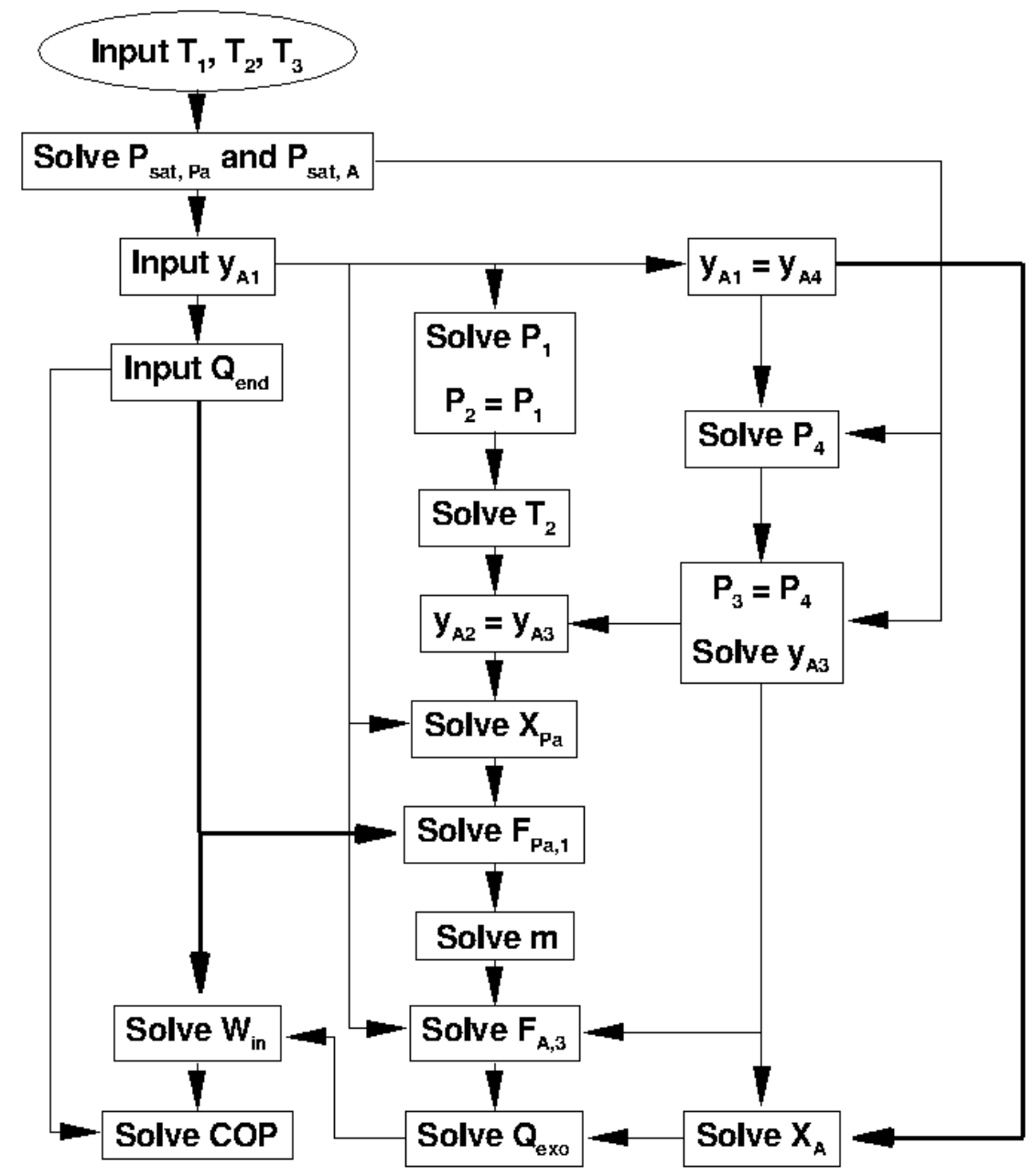

Figure 6: Paraldehyde chemical heat pump solution flow chart. 


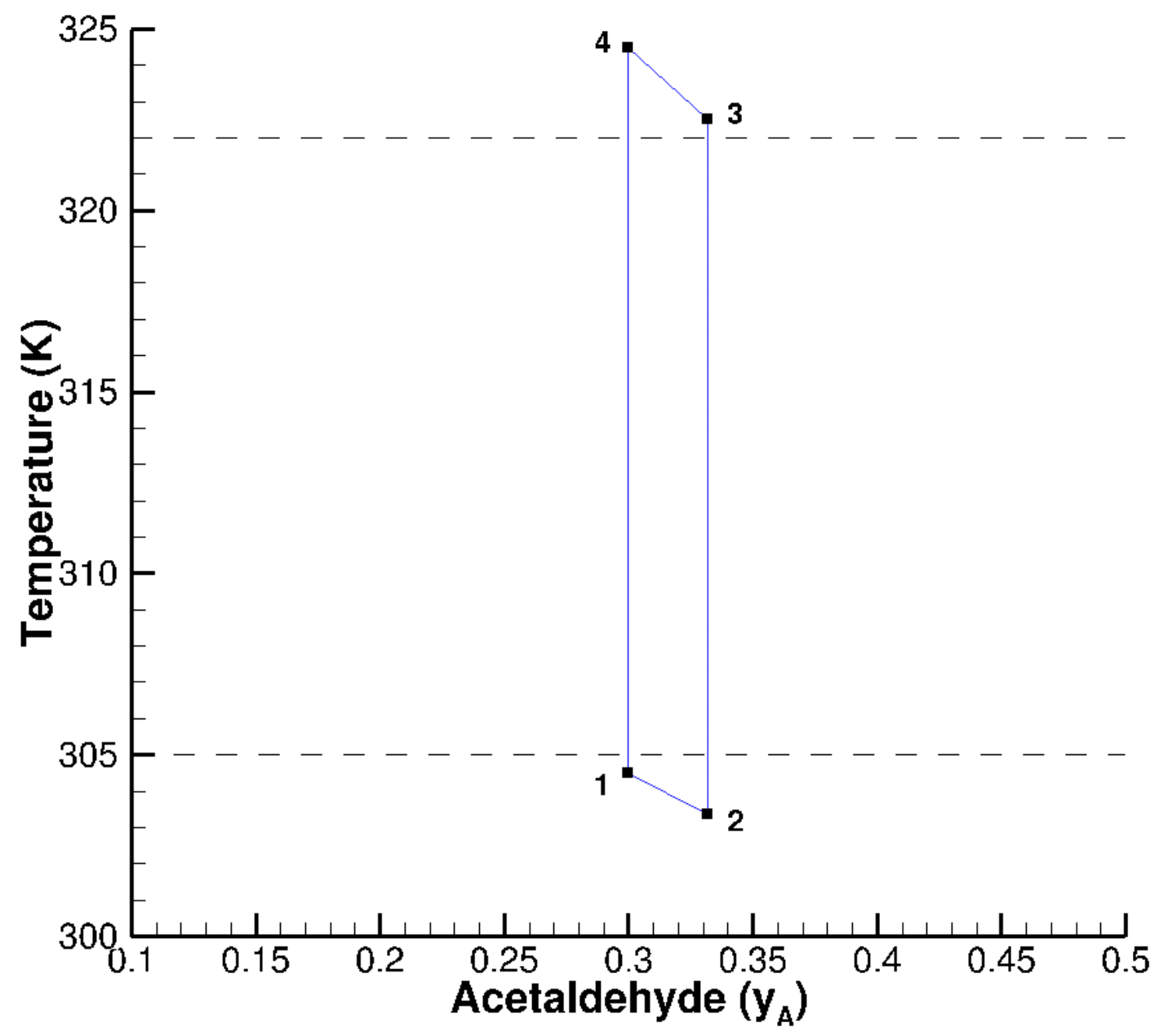

Figure 7: Temperature vs. mole fraction plot with 0.3 mole fraction of acetaldehyde at the endothermic reactor inlet. 


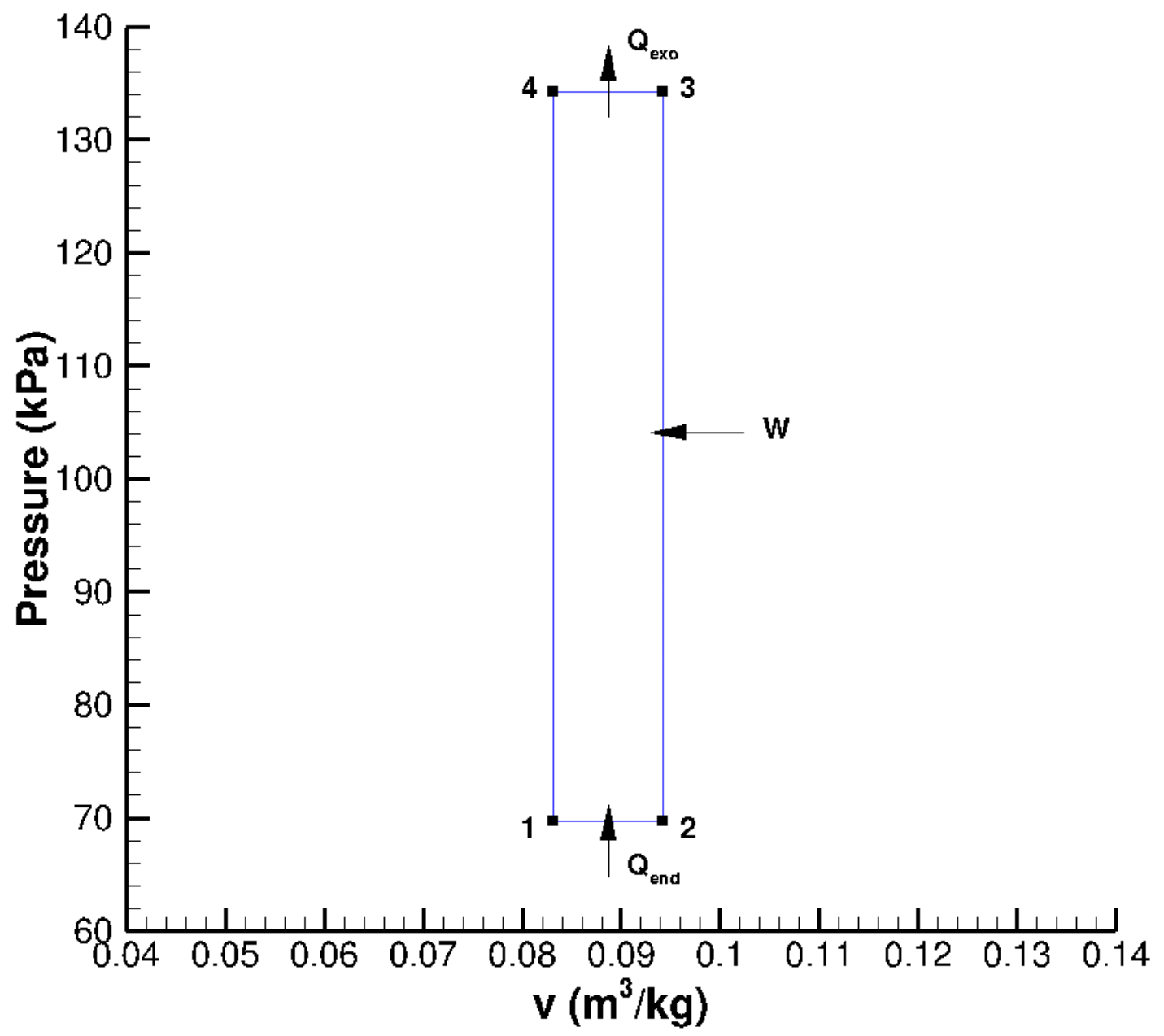

Figure 8: Pressure vs. specific volume with 0.3 mole fraction of acetaldehyde at the endothermic reactor inlet. 


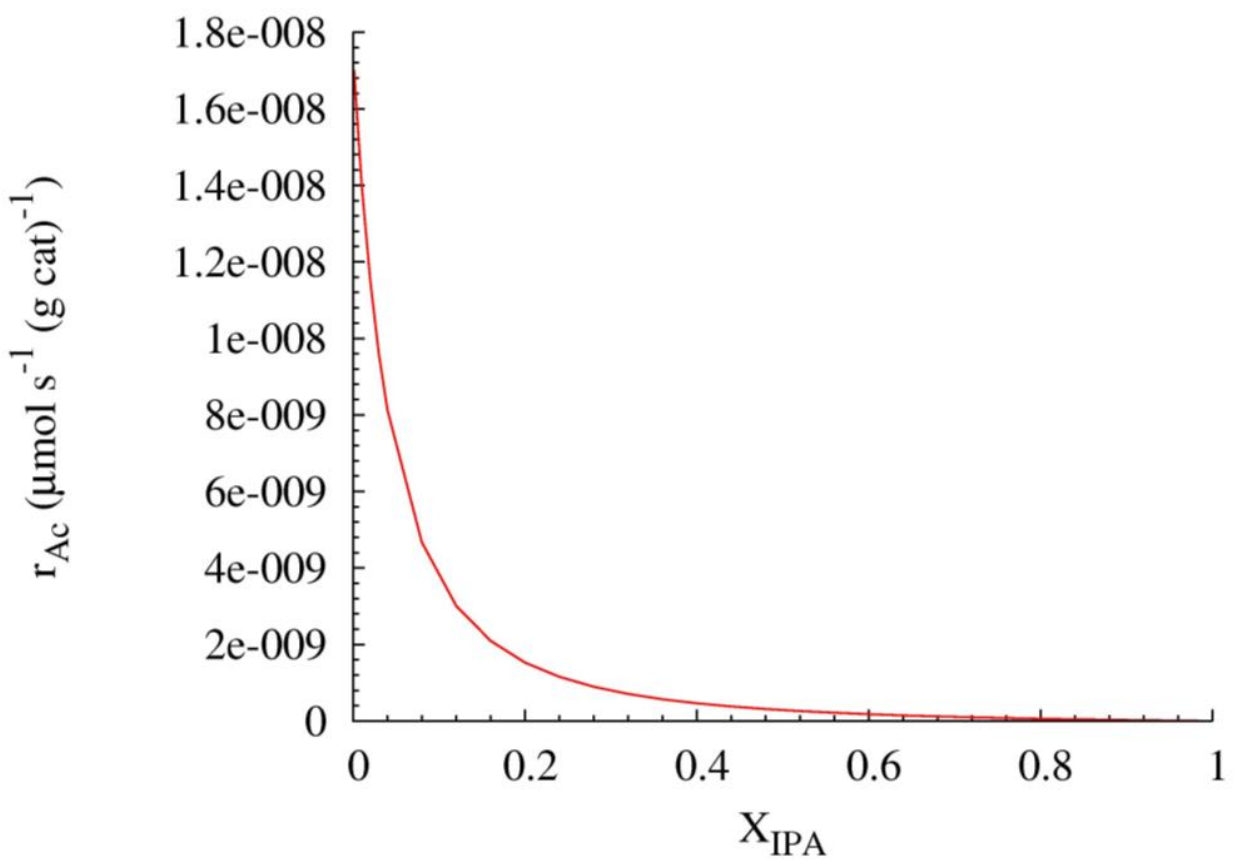

Figure 9: Rate of reaction as a function of 2-propanol conversion. 


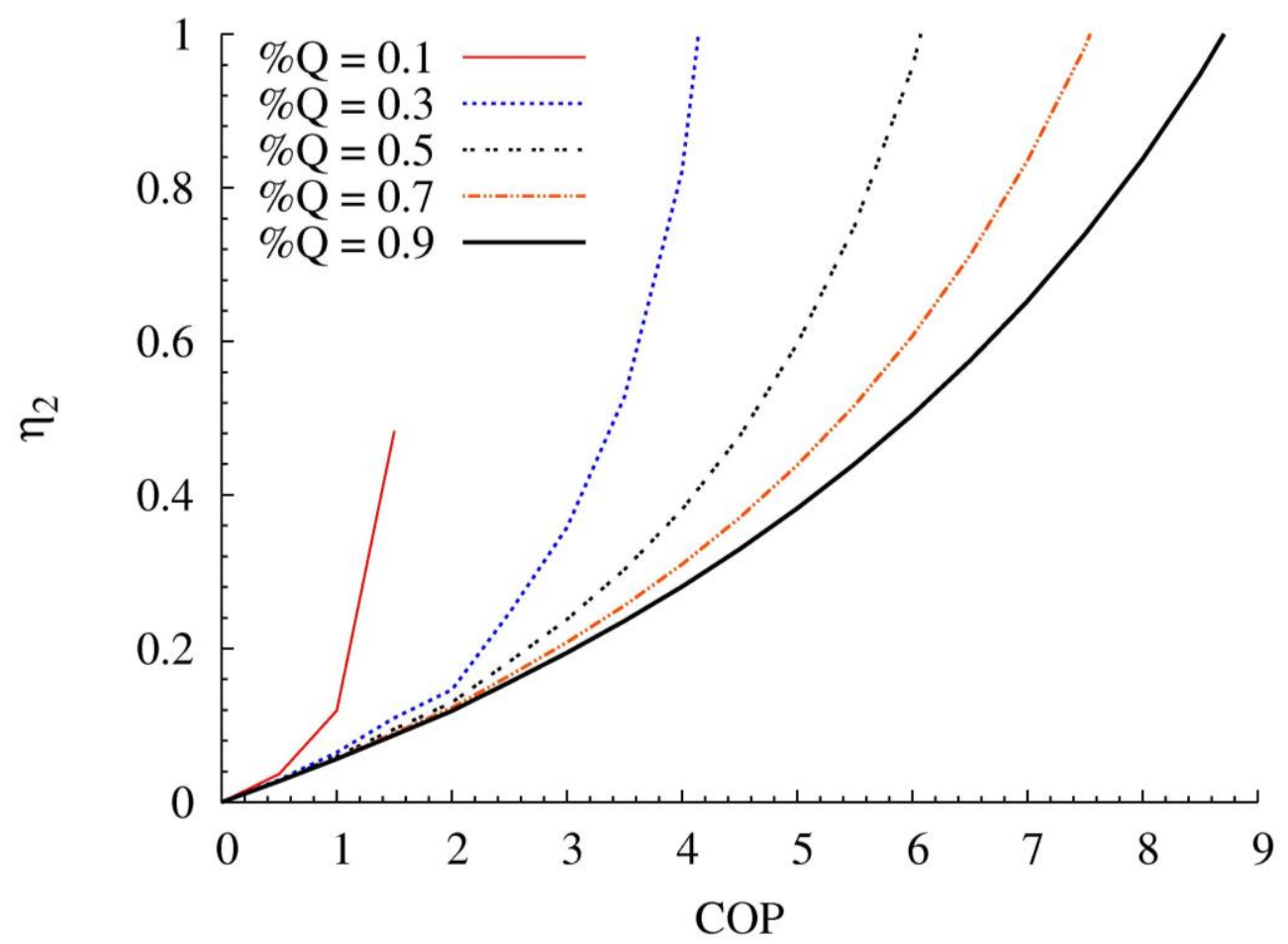

Figure 10: Exergy efficiency as a function of COP for different amounts of upgraded heat. 